Proc. of the 11 Int. School on Theoretical Physics Symmetry and Structural Properties of Condensed Matter, Rzeszów 2014

\title{
Numerical Stability of Solution of Recursion Relation for Ideal Quantum Gases Containing Finite Number of Harmonically Trapped Particles
}

\author{
T. PASZKIEWICZ ${ }^{a, *}$ AND S. WOLSKI ${ }^{b}$ \\ ${ }^{a}$ Retired from Faculty of Mathematics and Applied Physics, Rzeszów University of Technology, Rzeszów, Poland \\ ${ }^{b}$ Faculty of Mathematics and Applied Physics, Rzeszów University of Technology, \\ al. Powstańców Warszawy 6, PL-35959 Rzeszów, Poland
}

\begin{abstract}
The numerical stability of the solution of recursion relation for mean occupation numbers derived by Schönhammer for ideal Fermi gas trapped in 1D harmonic potential is studied. In low temperature region there exists a solution of this recursion relation. In high temperature region the iteration becomes unstable. In low and high temperature regions with growing number of particles the region of numerical instability diminishes.
\end{abstract}

DOI: 10.12693 /APhysPolA.128.204

PACS: 05.30.Fk, 03.75.Ss

\section{Introduction}

The achievement of the Bose-Einstein condensation (BEC) [1], degenerate Fermi gases [2, 3] was a remarkable attainment in experimental physics. The ultra-cold gases have been trapped magnetically in potential accurately approximated by harmonic oscillator wells. In the mentioned experiments ultra-cold atomic gases are isolated, i.e. do not exchange energy and matter with exterior and they contain mesoscopic number $N$ of atoms, much lower than the Avogadro number $N_{\mathrm{A}}\left(N \approx 10^{3}-10^{6}\right)$. In many experiments ultra-cold atomic gases are diluted, which makes the ideal gases fairly good approximation. This means that the physics of such ultra-cold gases should be described by the microcanonical approach to ideal quantum gases.

In the theoretical description of a system containing a finite number of particles, e.g. small metal particles (for example quantum dots and wires) $[4,5]$, and the fluorescence from a few electrons [6] one is not allowed to use the thermodynamic limit in which the particle number $N$ and the volume $V$ go to infinity with the density $N / V$ kept constant, leading to a continuum of single-particle levels. For the proper description of finite systems, the discrete nature of the single-particle spectrum has to be taken into account. These facts make impossible the standard use of grand-canonical Gibbs ensemble in thermodynamic limit.

However, a microcanonical theory of quantum gases is quite difficult. For an ideal Bose gas in a harmonic trap, Grossmann and Holthaus [7, 8] related the problem to the number-theoretical studies of partitions of an integer $q$ to the $m$ integers. The exact electron distribution in certain

\footnotetext{
* corresponding author; e-mail: paszkiewicz . tadeusz@gmail.com
}

small isolated systems was derived from the enumeration of the partitions of integers [9]. Kubasiak et al. [10] relate the problem of an ideal Fermi gas in a harmonic trap to the studies of partitions of $q$ into $m$ distinct integers. Schmidt and Schnack [11] related boson/fermion partition functions to symmetric polynomials.

Ligare $[12,13]$ pointed out that the harmonic oscillator potential used to model experiments on the BoseEinstein condensation of finite isolated atomic gases simplifies the numerical microcanonical analysis. As a rule the canonical theory of ideal quantum gases is simpler. It was proven that canonical and microcanonical descriptions of finite systems of quantum gases trapped in 1D harmonic traps are equivalent [14-16].

In this paper we report on the numerical stability of method of recursion relations applied in the frame of canonical theory to finite systems of ideal gases of fermions [17] trapped in 1D harmonic oscillators wells. After Shankar [18] we shall call these systems the gases of fermionic oscillators.

The case of bosons in such traps is discussed many in papers, e.g. [19, 20].

\section{Free energy and thermodynamic functions}

The energy spectrum of a particle in 1D harmonic well is non-degenerate and equidistant

$$
\varepsilon_{i}=i \Delta(i=1,2,3, \ldots) \text {. }
$$

In our calculations we shall ignore the zero point energy $\Delta / 2$, which plays no role in statistical physics. With the Fermi energy $\varepsilon_{\mathrm{F}}$ the level spacing $\Delta=\varepsilon_{\mathrm{F}} / N$.

We consider the gas of the Fermi oscillators in the frame of canonical ensemble. $T$ is temperature of this gas. The number of particles $N$ of the gas is fixed. The number of particles $N$ and the energy $E$ of the gas fulfill two obvious conditions

$$
N=\sum_{i=1}^{\infty} n_{i}, \quad E=\sum_{i=1}^{\infty}(i \Delta) n_{i},
$$


where $n_{i}=0,1$ is the occupation number of $i$-th oneparticle energy level $(i=1,2,3, \ldots)$. The first condition of (2) makes the calculation of the canonical partition function more difficult than the evaluation of the grand canonical partition function.

Let us write down the partition function $Z_{N}$ accounting for the condition imposed on the occupation numbers

$$
Z_{n}=\sum_{n_{1}=0}^{1} \sum_{n_{2}=0}^{1} \ldots \exp \left(-\sum_{i=1}^{\infty} \varepsilon_{i} n_{i} / \theta\right) \delta_{N, \sum_{j=1}^{\infty} n_{j}},
$$

where $\theta=k_{\mathrm{B}} T$ is the temperature of the bath in natural units.

Schönhammer [17] derived the recursion relation connecting the partition functions of systems of $N$ and $(N-1)$ particles

$$
Z_{n}=\frac{\mathrm{e}^{-N \Delta / \theta}}{1-\mathrm{e}^{-N \Delta / \theta}} Z_{N-1},
$$

with

$$
Z_{1}=\sum_{i=1}^{\infty} \mathrm{e}^{-\mathrm{i} \Delta / \theta}=\frac{\mathrm{e}^{-\Delta / \theta}}{1-\mathrm{e}^{-\Delta / \theta}}
$$

The ratio $Z_{N-1} / Z_{n}$ can be expressed by the chemical potential

$$
Z_{N-1} / Z_{n}=\mathrm{e}^{\mu_{n} / \theta} .
$$

From Eqs. (4), (5) it follows that:

$$
Z_{n}=\mathrm{e}^{-E_{n}^{(0)} / \theta} \prod_{m=1}^{n}\left(1-\mathrm{e}^{-m \Delta / \theta}\right)^{-1}
$$

where $E_{n}^{(0)}=\sum_{i=1}^{n} i \Delta=\Delta N(N-1) / 2$ is the ground state energy of the gas.

As one may expect, the obtained partition function has exactly the form of a partition function of $N$ uncoupled harmonic oscillators [21] with frequencies $\omega_{i}=i \Delta / \hbar$. The highest frequency is $\omega_{n}=N \Delta / \hbar=\varepsilon_{\mathrm{F}} / \hbar$.

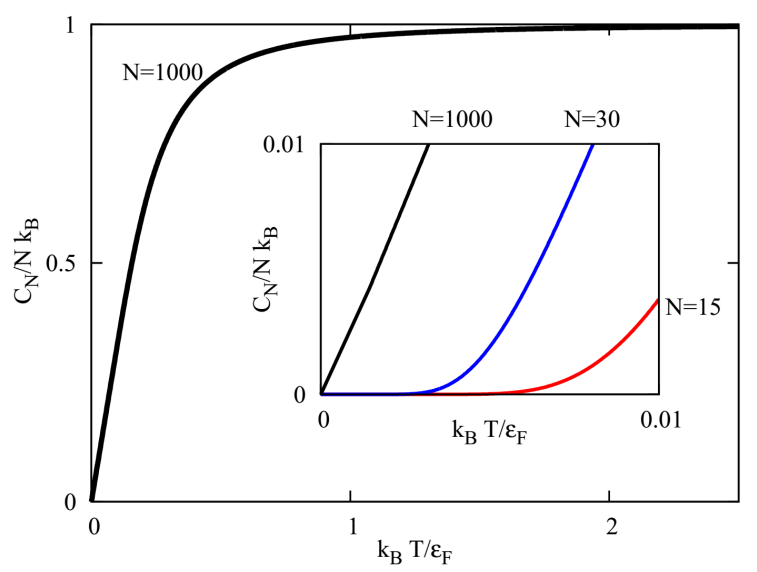

Fig. 1. Dependence of specific heat per particle $C_{n} / N$ on temperature for $N=1000$. In the inset $C_{n} / k_{\mathrm{B}} N$ vs. $\theta / \varepsilon_{\mathrm{F}}$ is shown for various number of particles $N$ for small ratio $\theta / \varepsilon_{F}$.

The knowledge of the partition function $Z_{n}$ allows one to calculate the free energy $F_{n}(\theta)$, the internal energy
$U_{n}(\theta)$, the heat capacity $C_{n}(\theta)$ :

$$
\begin{aligned}
& C_{n}(\theta)=k_{\mathrm{B}} \sum_{s=1}^{n} \frac{(m \Delta / \theta)^{2}}{\left(\mathrm{e}^{m \Delta / \theta}-1\right)^{2}} \mathrm{e}^{m \Delta / \theta}= \\
& k_{\mathrm{B}} \sum_{m=1}^{n} \frac{\left(m \varepsilon_{\mathrm{F}} / N \theta\right)^{2}}{\left(\mathrm{e}^{m \varepsilon_{\mathrm{F}} / N \theta}-1\right)^{2}} e e^{m \varepsilon_{\mathrm{F}} / N \theta},
\end{aligned}
$$

and the chemical potential $\mu_{n}(\theta)=\left[F_{n}(\theta)-F_{N-1}(\theta)\right]$ :

$$
\mu_{n}=\varepsilon_{\mathrm{F}}+\theta \ln \left(1-\mathrm{e}^{-\varepsilon_{\mathrm{F}} / \theta}\right) .
$$

In Fig. 1 it is visible that for mesoscopic number of particles at low temperatures the heat capacity is a linear function of temperature.

\section{Recursion relations for a gas of fermionic oscillators}

Now we shall study mean values of the occupation numbers $\left\langle n_{i}\right\rangle_{N}$ :

$$
\begin{aligned}
& \left\langle n_{i}\right\rangle_{n}= \\
& Z_{n}^{-1} \sum_{n_{1}=0}^{1} \sum_{n_{2}=0}^{1} \ldots n_{j} \exp \left(-\sum_{j=1}^{\infty} \varepsilon_{j} n_{j} / \theta\right) \delta_{N, \sum_{m=1}^{\infty} n_{m}} .
\end{aligned}
$$

Following [17] one obtains

$$
\left\langle n_{i}\right\rangle_{n}=\left(1-\left\langle n_{i}\right\rangle_{N-1}\right) \mathrm{e}^{-\left(\varepsilon_{i}-\mu_{n}\right) / \theta} .
$$

If $\left\langle n_{i}\right\rangle_{n}=\left\langle n_{i}\right\rangle_{N+1}$ the above formula leads to the FermiDirac distribution $\left\langle n_{i}\right\rangle_{\mathrm{F}}=\left[\mathrm{e}^{\left(\varepsilon_{i}-\mu\right) / \theta}+1\right]^{-1}$.

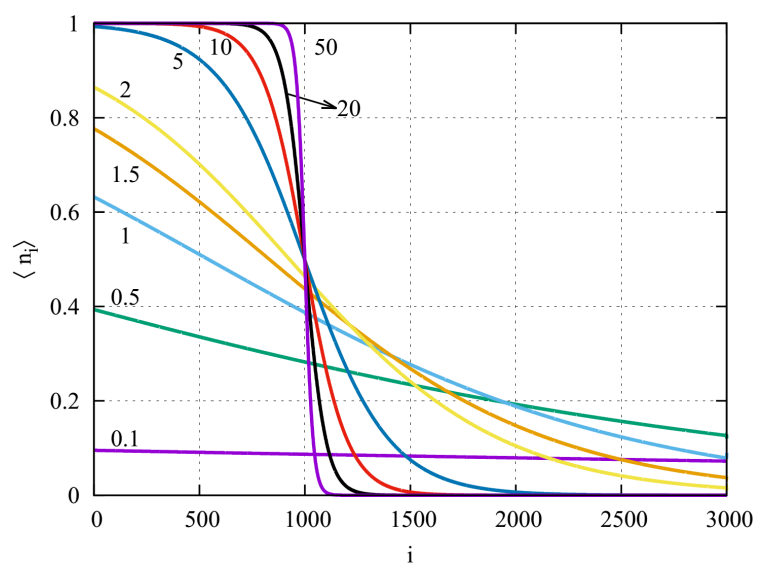

Fig. 2. Gas of fermionic oscillators contains $N=1000$ particles. Dependence of mean value of occupation numbers $\left\langle n_{i}\right\rangle_{n}$ on the number of the energy level for various values of the ratio $\varepsilon_{\mathrm{F}} / \theta$. Values of this ratio are indicated at the curves.

However, we shall study deviation from this function. For this end one uses the recursion relations. One of them is the "upward version" of it

$$
\left\langle n_{i+1}\right\rangle_{n}=a^{(\uparrow)}-b_{i}^{(\uparrow)}\left\langle n_{i}\right\rangle_{n},
$$

where

$$
a^{(\uparrow)}=\left(1-\mathrm{e}^{-\varepsilon_{\mathrm{F}} / \theta}\right),
$$




$$
b_{i}^{(\uparrow)}=\mathrm{e}^{-\varepsilon_{\mathrm{F}} / \theta}\left(\mathrm{e}^{\mathrm{i} \Delta / \theta}-1\right)=\mathrm{e}^{-\varepsilon_{\mathrm{F}} / \theta}\left(\mathrm{e}^{\mathrm{i} \varepsilon_{\mathrm{F}} / N \theta}-1\right) .
$$

The "upward version" of the recursion relation can be transformed to the "downward version" of it

$$
\left\langle n_{i}\right\rangle_{n}=a_{i}^{(\downarrow)}-b_{i}^{(\downarrow)}\left\langle n_{i+1}\right\rangle_{n},
$$

where

$$
\begin{aligned}
& a_{i}^{(\downarrow)}=\frac{\mathrm{e}^{\varepsilon_{\mathrm{F}} / \theta}-1}{\mathrm{e}^{\mathrm{i} \varepsilon_{\mathrm{F}} / N \theta}-1}, \\
& b_{i}^{(\downarrow)}=\frac{\mathrm{e}^{\varepsilon_{\mathrm{F}} / \theta}}{\mathrm{e}^{\mathrm{i} \varepsilon_{\mathrm{F}} / N \theta}-1} .
\end{aligned}
$$

Results of solution of Eqs. (9) and (10) are shown in Fig. 2.

\section{Examination of the stability of numerical calculations}

Using Eq. (9) we begin calculations with $\left\langle n_{1}\right\rangle_{n}=$ $\left[1-\exp \left(-\varepsilon_{\mathrm{F}} / \theta\right)\right]$. The upward iteration becomes numerically unstable for $i>i_{\max }$, where $i_{\max }$ depends on temperature and the number of particles. This means that procedure is stable for $i<i_{\max }$ (for $i$ below the full line in Fig. 3).

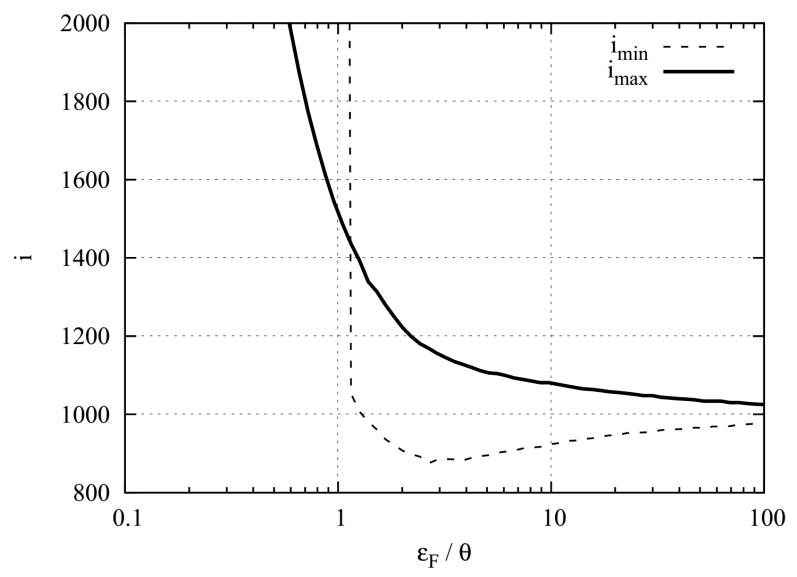

Fig. 3. Dependence of $i_{\max }$ and $i_{\min }$ on the inverse of temperature.

Using Eq. (10) one may begin calculations with $i=$ $(N+M)$. The value of $M$ is arbitrary, as long as $i>N$. The downward iteration becomes unstable for $i<i_{\text {min }}$, which also depends on temperature and $N$ (for $i$ above the dashed line in Fig. 3). For $\left(\varepsilon_{\mathrm{F}} / \theta\right)_{s}$ the lines in Fig. 3 intersect.

We conclude that for $\varepsilon_{\mathrm{F}} / \theta>\left(\varepsilon_{\mathrm{F}} / \theta\right)_{s}$, i.e. in the low temperature region, there exists solution of recursion relations (9) and (10). In the high temperature region where $\varepsilon_{\mathrm{F}} / \theta<\left(\varepsilon_{\mathrm{F}} / \theta\right)_{s}$ numerical calculations are unstable, hence the recursion relation has not solutions.

One may ask if the temperature region where numerical instability precludes numerical solution of the recursion relation depends on $N$. In Fig. 4 it is seen that for low temperatures (we chose $\theta=\varepsilon_{\mathrm{F}} / 10$ ) there exist $i$ obeying the inequality $i_{\min }<i<i_{\max }$. With growing $N i_{\min } \rightarrow i_{\max }$.

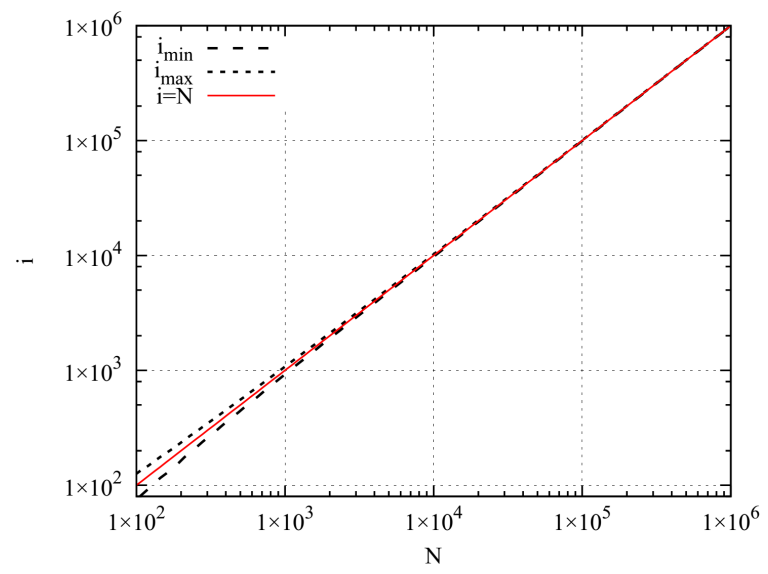

Fig. 4. Dependence of $i_{\max }$ and $i_{\min }$ on the number of particles $N$ for $\theta=\varepsilon_{\mathrm{F}} / 10$.

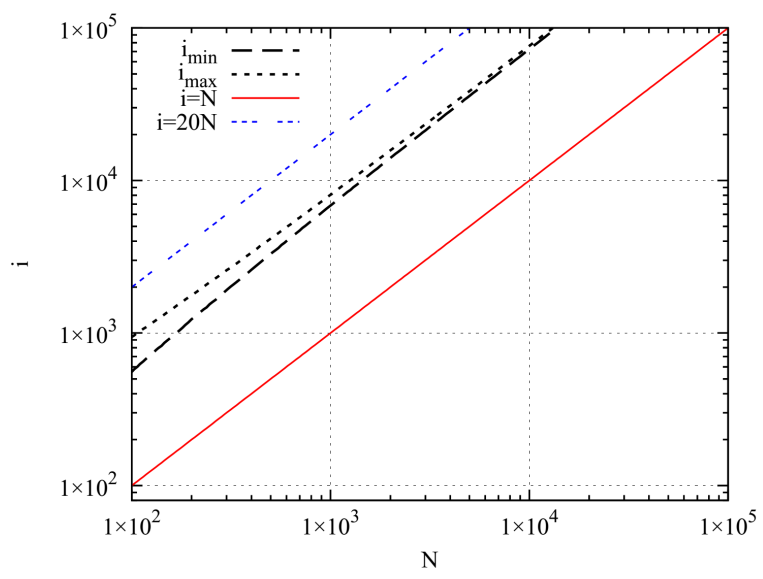

Fig. 5. Dependence of $i_{\max }$ and $i_{\min }$ on the number of particles $N$ for $\left(\varepsilon_{\mathrm{F}} / k_{\mathrm{B}} T\right)=0.1$.

Similar behavior is observed for high temperature region (cf. Fig. 5). For $\theta=10 \varepsilon_{F}$, i.e. in the high temperature region, $i_{\min } \rightarrow i_{\max }$ with growing $N$.

\section{References}

[1] L.P. Pitaevskii, S. Stringari, Bose-Einstein Condensation, Clarendon, Oxford 2003.

[2] L.P. Pitaevskii, S. Stringari, Bose-Einstein Condensation, Clarendon, Oxford 2003.

[3] S. Giorgini, L.P. Pitaevskii, S. Stringari, Rev. Mod. Phys. 80, 1215 (2008).

[4] R. Denton, B. Muhlschlegel, D.J. Scalapino, Phys. Rev. B 7, 3589 (1973).

[5] J. Arnaud, J.M. Boé, L. Chusseau, F. Philippe, Am. J. Phys. 67, 215 (1999).

[6] J. Arnaud, L. Chusseau, F. Philippe, Phys. Rev. B 62, 13482 (2000). 
[7] S. Grossmann, M. Holthaus, Phys. Rev. E 54, 3495 (1996).

[8] S. Grossmann, M. Holthaus, Chaos Solitons Fractals 10, 795 (1999).

[9] J.-M. Boé, F. Philippe, J. Comb. Theor. Series A 92, $173(2000)$

[10] A. Kubasiak, J.K. Korbicz, J. Zakrzewski, M. Lewenstein, Europhys. Lett. 72, 506 (2005).

[11] H.-J. Schmidt, J. Schnack, Am. J. Phys. 70, 53 (2002).

[12] M. Ligare, Am. J. Phys. 66, 185 (1998).

[13] M. Ligare, Am. J. Phys. 70, 76 (2002).

[14] M. Holthaus, E. Kalinowski, K. Kirsten, Ann. Phys. (New York) 270, 198 (1998).
[15] M.N. Tran, M.V.N. Murthy, R.K. Badhuri, Phys. Rev. E 63, 031105 (2001).

[16] F. Philippe, J. Arnoud, L. Chusseau, arXiv:math$\mathrm{ph} / 0211029 \mathrm{v} 1,2002$.

[17] K. Schönhammer, Am. J. Phys. 68, 1032 (2000)

[18] R. Shankar, Principles of Quantum Mechanics, Kluwer Academic, Plenum Publ., New York 2004.

[19] W.J. Mullin, J.P. Fernandez, Am. J. Phys. 71, 661 (2003).

[20] E.D. Trifonov, S.N. Zagoulaev, Phys.-Usp. 53, 83 (2010).

[21] N.W. Ashcroft, N.D. Mermin, Solid State Physics, Holt, Rinehart and Winston, New York 1976. 